\title{
A hydrogel-endothelial cell implant mimics infantile hemangioma: modulation by survivin and the Hippo pathway
}

Masayuki Tsuneki ${ }^{1,3}$, Steven Hardee ${ }^{1}$, Michael Michaud', Raffaella Morotti', Erin Lavik ${ }^{2}$ and Joseph A Madri ${ }^{1}$

Microvascular endothelial cells cultured in three-dimensional hydrogel scaffolds form a network of microvessel structures when implanted subcutaneously in mice, inosculate with host vessels, and over time remodel into large ectatic vascular structures resembling hemangiomas. When compared with infantile hemangiomas, similarities were noted, including a temporal progression from a morphological appearance of a proliferative phase to the appearance of an involuted phase, mimicking the proliferative and involutional phases of infantile hemangioma. Consistent with the progression of a proliferative phase to an involuted phase, both the murine implants and human biopsy tissue exhibit reduced expression of Ajuba, YAP, and Survivin labeling as they progressed over time. Significant numbers of CD45+, CD11b+, Mac3+ mononuclear cells were found at the 2-week time point in our implant model that correlated with the presence of CD45+, CD68+ mononuclear cells observed in biopsies of human proliferative-phase hemangiomas. At the 4-week time point in our implant model, only small numbers of CD45+ cells were detected, which again correlated with our findings of significantly diminished CD45+, CD68+ mononuclear cells in human involutional-phase hemangiomas. The demonstration of mononuclear cell infiltration transiently in the proliferative phase of these lesions suggests that the vascular proliferation and/or regression may be driven in part by an immune response. Gross and microscopic morphological appearances of human proliferative and involutional hemangiomas and our implant model correlate well with each other as do the expression levels of Hippo pathway components (Ajuba and YAP) and Survivin and correlate with proliferation in these entities. Inhibitors of Survivin and Ajuba (which we have demonstrated to inhibit proliferation and increase apoptosis in murine hemangioendothelioma cell tissue culture) may have potential as other beneficial treatments for proliferating infantile hemangiomas. This implant model may have potential as a modest through-put screen for testing and development of therapeutics targeted at the proliferative phase of infantile hemangiomas, reducing the subsequent postinvolutional scarring or deformities sometimes associated with these lesions.

Laboratory Investigation (2015) 95, 765-780; doi:10.1038/labinvest.2015.61; published online 11 May 2015

Implantation of a variety of cell types in three-dimensional scaffolds (biological and synthetic biocompatible) has been used extensively in a wide range of settings, including attempts to improve structure and function of mesenchymal scaffolds in a variety of soft and hard tissue applications, ${ }^{1,2}$ enhancement of vasculature following injury, ${ }^{3}$ enhancing repair/recovery of parenchymal tissue following injury, ${ }^{4}$ and production and delivery of soluble proteins. ${ }^{5}$ In addition, implantation of cells in three-dimensional scaffolds has been used to enhance survivability ${ }^{6}$ and investigate basic physiological and pathophysiological processes, including angiogenesis, ${ }^{7,8}$ cell migration and invasion, ${ }^{9}$ differentiation, ${ }^{10}$ and neoplasia. ${ }^{11}$ Using both biological (collagen and other ECM components) and biocompatible (hydrogels) scaffolds we have demonstrated the efficacy of employing scaffolds in combination with selected cell types as protein delivery vehicles ${ }^{5}$ as well as in several angiogenesis and neural progenitor studies. ${ }^{10,12,13}$ In this report, we found that when implanted subcutaneously, immortalized murine microvascular endothelial cells dispersed in a hydrogel scaffold undergo marked proliferation and became vascularized. Interestingly, over time (4 weeks) these implants developed a lumpy, bulging appearance, resembling clusters of

'Department of Pathology, Yale University School of Medicine, New Haven, CT, USA and ²Department of Bioengineering, Case Western Reserve University, Cleveland, OH, USA

Correspondence: Professor JA Madri, MD, PhD, Department of Pathology, Yale University School of Medicine, Lauder Hall, Room L115, 310 Cedar Street, New Haven, CT 06510, USA. 
dilated vessels. These morphological changes that have occurred over time resemble the morphological changes observed in infantile hemangiomas as they progress from the proliferative to the involutional phase. In this report, we also have documented the dynamic presence of immune cells and the expression of selected proteins known to modulate proliferation and survival in a wide range of cell types, including endothelia in both the implants and infantile hemangiomas. Specifically, we illustrate the changes in macrophage presence and expression of Ajuba, Survivin, and YAP in both the clinical material and the murine implants and suggest that the murine implants may serve as a useful animal model of this entity, facilitating the development of therapeutics focused on blunting the proliferative phase and reducing the postinvolutional scarring that sometimes accompanies the regression of these lesions.

\section{MATERIALS AND METHODS \\ Cell Culture}

Murine brain endothelial cells (BECs) were isolated from cerebral microvessels of C57BL/6 wild-type mice (WT-BEC) (The Jackson Laboratory, Bar Harbor, ME, USA) as shown previously. ${ }^{14-16}$ WT-BEC was cultured on $1.5 \%$ gelatin (Cat. No. G8-500, Thermo Fisher Scientific, Waltham, MA, USA) coated plates in BEC media (Dulbecco's Modified Eagle's Medium (DMEM) with High Glucose (Life Technologies, Grand Island, NY, USA) containing 10\% FBS, 2 mM L-glutamine, $0.1 \mathrm{mM}$ nonessential amino acids, $1 \mathrm{mM}$ sodium pyruvate, $10 \mathrm{mM}$ HEPES (pH 7.4), $10^{-5} \mathrm{M} \beta$-mercaptoethanol, $100 \mathrm{U} / \mathrm{ml}$ penicillin, and $100 \mu \mathrm{g} / \mathrm{ml}$ streptomycin (Life Technologies)) in $8 \% \mathrm{CO}_{2}$ at $37^{\circ} \mathrm{C} .{ }^{9,16}$ Cells were used in passage 22 and cultured under normoxic $\left(20 \% \mathrm{O}_{2}\right)$ condition.

\section{Dispersing Endothelial Cells in Poly(Ethylene Glycol)/ Poly(L-Lysine) (PEG/PLL) Hydrogels}

We performed testing for endotoxin on the polymer precursors and have not observed indications consistent with endotoxin contamination. The inflammation we observed appears to be related to the cell types used. When these same materials are used with other cell types, we do not see similar infiltration of inflammatory cells subcutaneously and in the spinal cord. ${ }^{3,10,12,17,18}$ Irgacure 2959 initiator was dissolved in BEC media at a concentration of $5 \mathrm{mg} / \mathrm{ml}$ and filtered with $0.22-\mu \mathrm{m}$ sterile syringe filter (Cat. No. SLGP033RS, MillexGP Syringe Filter Unit, $0.22-\mu \mathrm{m}$ pore size hydrophilic polyethersulfone membrane; Merck Millipore, Billerica, MA, USA) before crosslinking. PEG/PLL macromers ${ }^{10,17-19}$ were dissolved at a concentration of $10 \%(\mathrm{w} / \mathrm{v})$ in the Irgacure 2959 initiator solution and mixed with WT-BEC at a concentration of $2.5 \times 10^{6}$ cells $/ \mathrm{ml}$. Aliquots of endothelial cells mixed with the macromer solution was placed under UV light for $10 \mathrm{~min}$ at $6 \mathrm{~mW} / \mathrm{cm}^{2}$, which elicited formation of the gels with endothelial cells dispersed throughout.

\section{Surgical Procedures}

Ten 12-week-old C57 black mice (C57BL/6) (The Jackson Laboratory) were used for the subcutaneous implantation studies. Each mouse was anesthetized with an intra-peritoneal injection of ketamine $(100 \mathrm{mg} / \mathrm{kg})$ and xylazine $(20 \mathrm{mg} / \mathrm{kg})$. An incision of $10 \mathrm{~mm}$ in length was made through the skin overlying the thoracic spine, and subcutaneous pockets were created bilaterally by blunt dissection under the skin. A round hydrogel disk ( $5 \mathrm{~mm}$ diameter) was implanted in each mouse, one in each pocket. The incision was closed with surgical clips, and the mice were maintained on a heating pad until they regained mobility. Following euthanasia, five Hydrogel implants were retrieved at 2 and 4 weeks, respectively, after implantation and fixed in 5\% paraformaldehyde in $50 \mathrm{~mm}$ HEPES buffer ( $\mathrm{pH}$ 7.3) overnight for histological analysis. All surgical procedures were approved by the Animal Care and Use Committees of Yale University (Animal Protocol No. 07366).

\section{Histology}

After fixation, excised implants were incubated in $15 \%$ and $30 \%$ Sucrose/PBS (pH 7.4) solutions sequentially overnight and embedded in PolyFreeze cryostat embedding medium (Cat. No. 19636, Polysciences, Warrington, PA, USA). Ten- $\mu \mathrm{m}$-thick sections were generated using a Cryotome FSE Cryostat (Thermo Fisher Scientific). Frozen sections were stained with Hematoxylin \& Eosin (H\&E) using Dako Mayer's Hematoxylin (Lillie's Modification) Histological Staining Reagent (Cat. No. S3309, DAKO, Carpinteria, CA, USA) and Dako Eosin (Cat. No. CS701, DAKO) for histopathological analysis.

\section{Antibodies}

Antibodies were directed against mouse CD31 (PECAM-1) (affinity purified SL-4), raised in rabbit and purified as described elsewhere. ${ }^{20}$ Rat monoclonal antibodies against mouse CD31 (clone: MEC13.3) (Cat. No. 550274), CD45 (30-F11) (Cat. No. 550539), CD11b (M1/70) (Cat. No. 550282), Mac3 (M3/84) (Cat. No. 550292), and VE-cadherin (VEC) (11D4.1) (Cat. No. 550548) were purchased from BD Biosciences (San Jose, CA, USA). Rabbit polyclonal antibodies against Ki67 (Cat. No. ab15580) were purchased from Abcam (Cambridge, MA, USA). Rabbit monoclonal antibodies against Survivin (71G4B7) (Cat. No. 2808), YAP (D24E4) (Cat. No. 8418), and phospho-YAP (Ser127) (D9W2I) (Cat. No. 13008) (P-YAP) were purchased from Cell Signaling Technology (Danvers, MA, USA). A mouse monoclonal antibody against glucose transporter 1 (GLUT-1) (SPM498) (Cat. No. ab40084) was purchased from Abcam. Mouse monoclonal antibodies against human CD31 (JC70A) (Cat. No. M0823), a CD45 (2B11+PD7/26) (Cat. No. M0701), and a monoclonal mouse anti-human CD68 (PG-M1) (Cat. no. IR61361) were purchased from DAKO. Rabbit polyclonal antibodies against human Ajuba (Cat. No. HPA006171) were purchased from Sigma-Aldrich (Saint Louis, MO, USA). 
A rabbit polyclonal against human GLUT-1 (Cat. No. 1128504 A) was purchased from Cell Marque (Rocklin, CA, USA).

\section{Immunofluorescence}

To prevent non-specific protein binding, frozen tissue sections were incubated with $5 \%$ normal goat serum (Cat. No. 005-000-121, Jackson ImmunoResearch Laboratories, West Grove, PA, USA) in PBS ( $\mathrm{pH}$ 7.4) containing 0.05\% Triton X-100 (T-PBS) for $1 \mathrm{~h}$ at room temperature. After blocking, sections were incubated with primary antibodies (CD31, CD45, CD11b, Mac3, and VEC diluted at 1:10 in T-PBS; CD31 (SL-4), YAP, and GLUT-1 (SPM498), diluted at 1:200; Survivin, diluted at 1:400; Ki67, diluted at 1:500; and phospho-YAP, diluted at 1:2000 over night at $4{ }^{\circ} \mathrm{C}$ and further with secondary antibodies (Alexa Fluor 488 or 594-conjugated goat anti-rabbit $\operatorname{IgG}(\mathrm{H}+\mathrm{L})$ or anti-rat $\operatorname{IgG}(\mathrm{H}+\mathrm{L})$ (Life Technologies)), diluted at 1:100 in T-PBS for $2 \mathrm{~h}$ at room temperature. Following three T-PBS washes, slides were coverslipped with Dako Fluorescence Mounting Medium (Cat. No. S3023, DAKO). Digital fluorescence images and phase-contrast microscopic photos were captured on an Olympus IX71 inverted microscope equipped with a MicroFire camera and PictureFrame 1.0 software for Macintosh (OPTRONICS, Goleta, CA, USA) and Photoshop CS2 software (Adobe, San Jose, CA, USA) on a Windows 7 computer.

\section{Clinical Cases}

Representative proliferating- and involuting-phase cutaneous infantile hemangioma cases were collected for the present study from the Surgical Pathology files of the Yale University School of Medicine. The surgical samples were fixed in $10 \%$ formalin, processed routinely, and embedded in paraffin. Serial sections cut at $5 \mu \mathrm{m}$ from paraffin blocks were used for
H\&E staining and immunohistochemistry. Briefly, a retrospective search of the pathology database was performed to identify cases of vascular tumors with the diagnosis of hemangioma. Over a 10-year period, a total of 58 cases were retrieved with the diagnosis. The original $\mathrm{H} \& \mathrm{E}$ slides were reviewed in a blinded manner by three pathologists (one pathologist was aware of the diagnosis on record) to determine whether there was concordance between the archived diagnosis and the diagnosis by the pathologists involved in this study. Out of these 58 cases, 13 cases were selected by consensus review, comprised of 6 infantile hemangiomas in the proliferative phase and 7 in the involutional phase. The selection was also partially based on the presence of sufficient tissue to perform additional immunohistochemical and microarray studies. Following immunohistochemical staining, the slides were reviewed in a blinded manner from the original diagnosis by all the pathologists. Additional details regarding the cases are shown in Table 1. The study was approved by the institution's IRB (Institutional Review Board).

\section{Tissue Microarray}

Thirteen representative proliferating- and involuting-phase infantile hemangiomas were collected from the Surgical Pathology files of the Yale University School of Medicine. All cases were reviewed using clinical records and conventional H\&E staining slides before selecting typical doubleredundant histopathological areas $(6 \mathrm{~mm}$ diameters, each) for the tissue microarray. Paraffin-embedded tissue microarray slides were generated by the Yale University Pathology Department Tissue Services (YPTS) and used for immunohistochemistry.

Table 1 Summary of the clinical cases assayed

\begin{tabular}{|c|c|c|c|c|c|c|}
\hline Case no. & Age@Bx & Gender & Tissue type & Site & H\&E diagnosis & Surv/Hippo analysis \\
\hline 1 & 1 year & M & Soft tissue & Chest & Juvenile/infantile capillary hemangioma, proliferative phase & Proliferating phase \\
\hline 2 & 7 months & $\mathrm{F}$ & Soft tissue & Axilla & Juvenile/infantile capillary hemangioma, proliferative phase & Proliferating phase \\
\hline 3 & 1 year & F & Skin & Scalp & Involuting infantile capillary hemangioma & Proliferating phase \\
\hline 4 & 3 months & M & Skin & Scalp & Infantile capillary hemangioma, proliferative phase & Proliferating phase \\
\hline 5 & 1 year & F & Skin & Shoulder & Infantile hemangioma, proliferative phase & Proliferating phase \\
\hline 6 & 1 year & F & Skin & Lip & Infantile hemangioma, proliferative phase & Proliferating phase \\
\hline 7 & 3 years & F & Soft tissue & Axilla & Juvenile/infantile hemangioma, proliferative phase & proliferating phase \\
\hline 8 & 1 year & M & Soft tissue & Forehead & Infantile/juvenile capillary hemangioma involutive phase & Transitional phase \\
\hline 9 & 1 year & $\mathrm{F}$ & Soft tissue & Forehead & Infantile hemangioma, involutive phase & Transitional phase \\
\hline 10 & 3 years & F & Skin & Eye & Juvenile/infantile capillary hemangioma, involutive phase & Involuting phase \\
\hline 11 & 3 years & $\mathrm{F}$ & Skin & Lip & Juvenile/infantile capillary hemangioma, involutive phase & Involuting phase \\
\hline 12 & 3 years & M & Skin & Lip & Juvenile/infantile capillary hemangioma, involutive phase & Involuting phase \\
\hline 13 & 7 years & $\mathrm{F}$ & Soft tissue & Forearm & Juvenile/infantile capillary hemangioma, involutive phase & Involuting phase \\
\hline
\end{tabular}


a
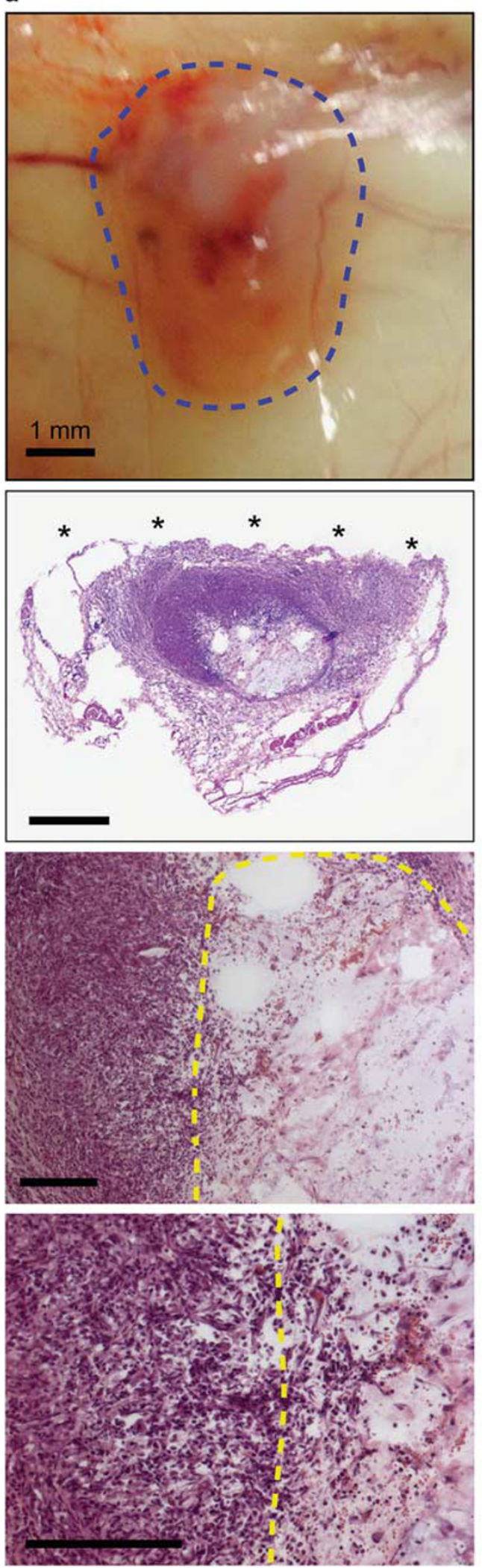

b
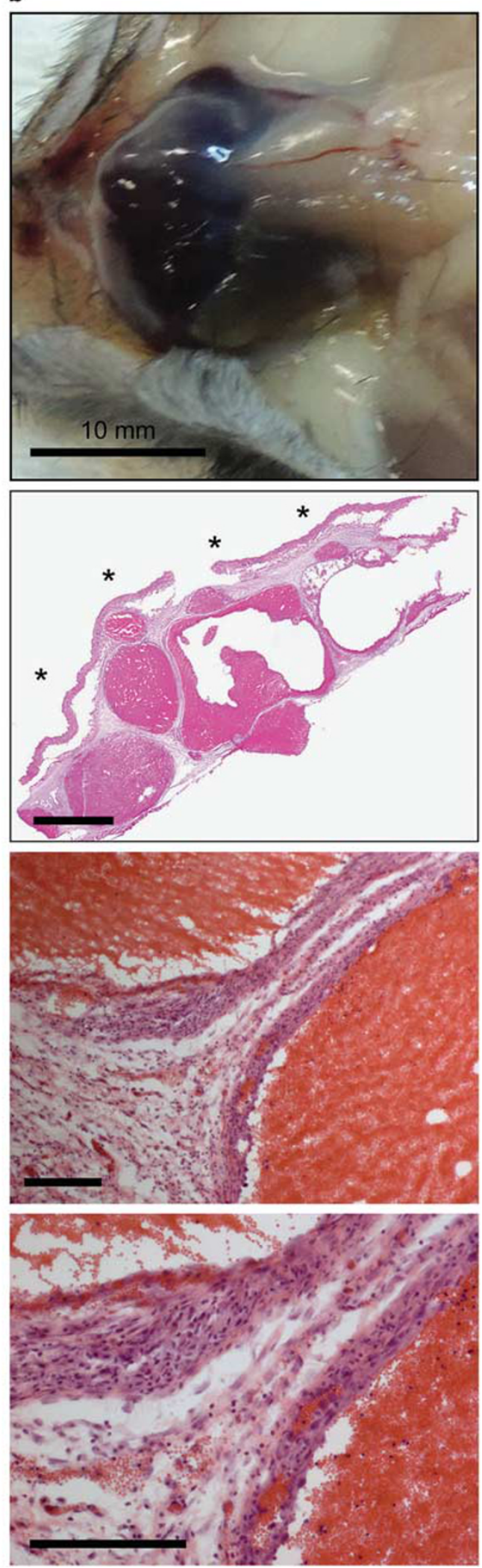


\section{Immunohistochemistry}

Immunohistochemistry was performed using the Dako EnVision+Dual Link System-HRP (DAKO). For antigen retrieval, sections were heated in citric acid buffer ( $\mathrm{pH}$ 6.0) at $100^{\circ} \mathrm{C}$ for $10 \mathrm{~min}$ using Lab Vision PT Module (Thermo Fisher Scientific). The sections were treated with $0.3 \%$ hydrogen peroxide in methanol for $30 \mathrm{~min}$ at room temperature to block endogenous peroxidase activity and incubated with 5\% normal goat serum in T-PBS for $1 \mathrm{~h}$ at room temperature to block non-specific protein-binding sites. They were then incubated at $4{ }^{\circ} \mathrm{C}$ with the primary antibodies diluted at $1: 40$ (anti-human CD31), 1:50 (anti-Ajuba and CD45), 1:200 (anti-YAP), 1:400 (anti-Survivin), 1:2 (anti-GLUT1), and 1:16 (anti-CD68) in T-PBS. After overnight incubation, the sections were incubated with EnVision reagents for $1 \mathrm{~h}$ at room temperature and treated with Dako Liquid DAB+ Substrate Chromogen System (Cat. No. K3468, DAKO) to visualize the reaction products. Sections were counterstained with Dako Mayer's Hematoxylin (Lillie's Modification) Histological Staining Reagent (DAKO). Digital immunohistochemical images were captured on an Olympus IX71 inverted microscope equipped with a MicroFire camera and PictureFrame 1.0 software for Macintosh (OPTRONICS) and Photoshop CS2 software (Adobe) on a Windows 7 computer.

\section{RESULTS \\ Implanted Endothelial Cell Hydrogel Scaffolds Exhibit Inosculation and Dynamic Remodeling Over Time}

Following subcutaneous implantation, all the three-dimensional endothelial cell containing hydrogel scaffolds elicited ingrowth of host vessels and inosculation with tube-like structures within the scaffolds, exhibiting a vascularized phenotype (Figure 1a, upper panel). Additionally, a robust inflammatory infiltrate mainly composed of mononuclear cells was noted infiltrating the implant at the 2-week time point (Figure 1a, second, third, and fourth panels). At the 4-week time point, the implants took on a bulging, bumpy appearance with a deep red-purple color (Figure 1b, upper panel). Large ectatic blood-filled structures were noted, separated by connective tissue septa containing several microvascular structures and a paucity of infiltrated immune cells (Figure 1b, second, third, and fourth panels).

\section{Implants Exhibit Dynamic Immune Cell Infiltration in the Peri-Implant Area Over Time}

At the 2-week time point, immunofluorescence staining with CD31 confirmed the robust neovascularization in periimplant areas elicited by the implant (Figure 2a, left column). Double staining with CD45, CD11b, and Mac3 (Figure 2a, (middle column, first, second, and third rows, respectively) revealed an intense labeling of mononuclear immune cells throughout the granulation tissue comprising the periimplant area. Merged images of CD31 and CD45 and CD11b and Mac3 (Figure 2a, left column) illustrate immune cell infiltration in close proximity to the microvasculature. High power insets of the merged images reveal an intimate association between the vasculature and the mononuclear immune cells.

At the 4-week time point, there was considerable remodeling of the implants. Specifically, ectatic blood-filled spaces were lined by endothelial cells and the intervening connective tissue septa exhibited a reduced density of microvasculature and a dramatic reduction in mononuclear immune cell infiltration. Occasional immune cells were noted adherent to and in close proximity with the endothelial cells lining the blood-filled spaces as evidenced by CD31 (Figure 2b, left column), CD45, and CD11b (Figure 2b, middle column) double staining. Merged images (Figure 2b, right column) further illustrate this co-localization.

\section{Proliferative Pathway Components are Expressed in a Dynamic Manner in and Around the Implants Over Time} In order to investigate the potential drivers of the early transient proliferative response in the implant tissue, we stained the tissues with CD31, VE-cadherin, Ki67, Survivin, YAP, and phospho-YAP. At the 2-week time point, immunoco-localization with CD31 (Figure 3) and VE-cadherin (first row) illustrates their co-localization in peri-implant areas (left side of the yellow dashed line) but a lack of VE-cadherin with CD31-positive vessels deeper within the implant (right of the yellow dashed line, circled endothelial cells), indicative of a

Figure 1 Macroscopic images and histopathology of subcutaneous hydrogel implants for 2 and 4 weeks. (a) The macroscopic image of hydrogel implanted for 2 weeks illustrates the apparent vascularization of the hydrogel implant (blue dashed line), as evidenced by its pinkish color, and a large feeder vessel at 10 O'clock and a smaller ones at 8 and 11 O'clock and running along the border of the implant from 2 to 5 O'clock. (Left panel, top row). Scale bar, $1 \mathrm{~mm}$. Low-power H\&E cross-section images of this tissue (left panel, second row from top) reveal a circumscribed area representing the implant, which has been partially infiltrated with inflammatory cells and granulation tissue that is also present surrounding the implant. Asterisks denote the skin surface. Scale bar, $1 \mathrm{~mm}$. Intermediate- (left panel, third row) and high-power (left panel, fourth row) images illustrate the ingrowth of inflammatory cells and granulation tissue into the implant proper. The yellow dotted lines represent the borderline between hydrogel and granulation tissue. Scale bars, $200 \mu \mathrm{m}$. (b) In contrast to the macroscopic appearance of the 2-week implants, the macroscopic image of a representative hydrogel implant after 4 weeks of implantation reveals a dark purple lobulated globular structure with occasional feeder vessels coursing toward and through it. Low-power H\&E cross-section images of this tissue (right panel, second row) reveals the formation of several large ectatic blood-filled vascular spaces in the dermis, underlying the surface skin. These hemangioma-like lesions exhibited clear margins. Asterisks denote the skin surface. Scale bar, $10 \mathrm{~mm}$. Intermediate- (right panel, third row) and high-power (right panel, fourth row) images illustrate fibrovascular cords forming the walls and separating the ectatic blood-filled vascular spaces. Also noted, in contrast to the 2-week implants, was a paucity of inflammatory infiltrate. Scale bars, $200 \mu \mathrm{m}$. 

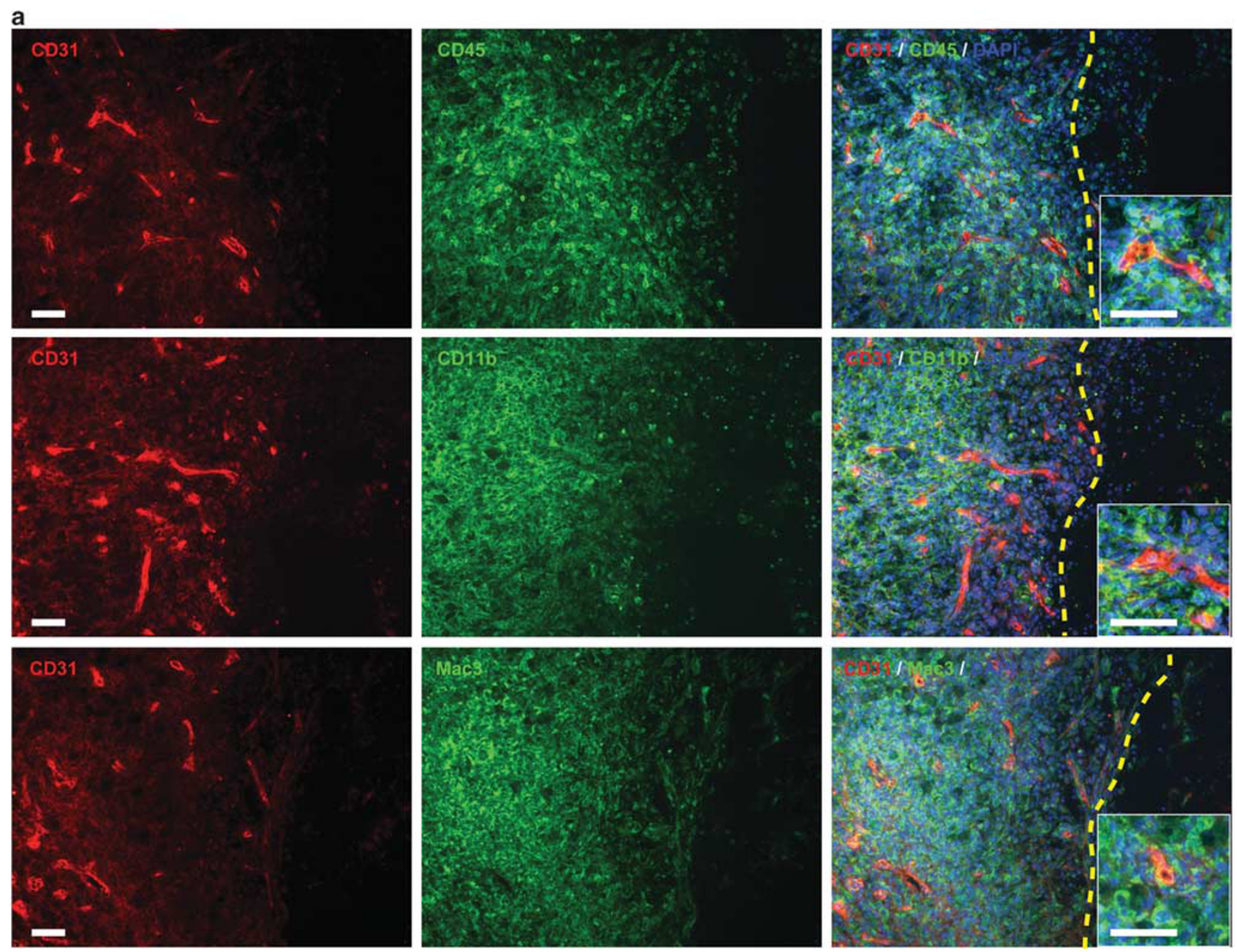

b
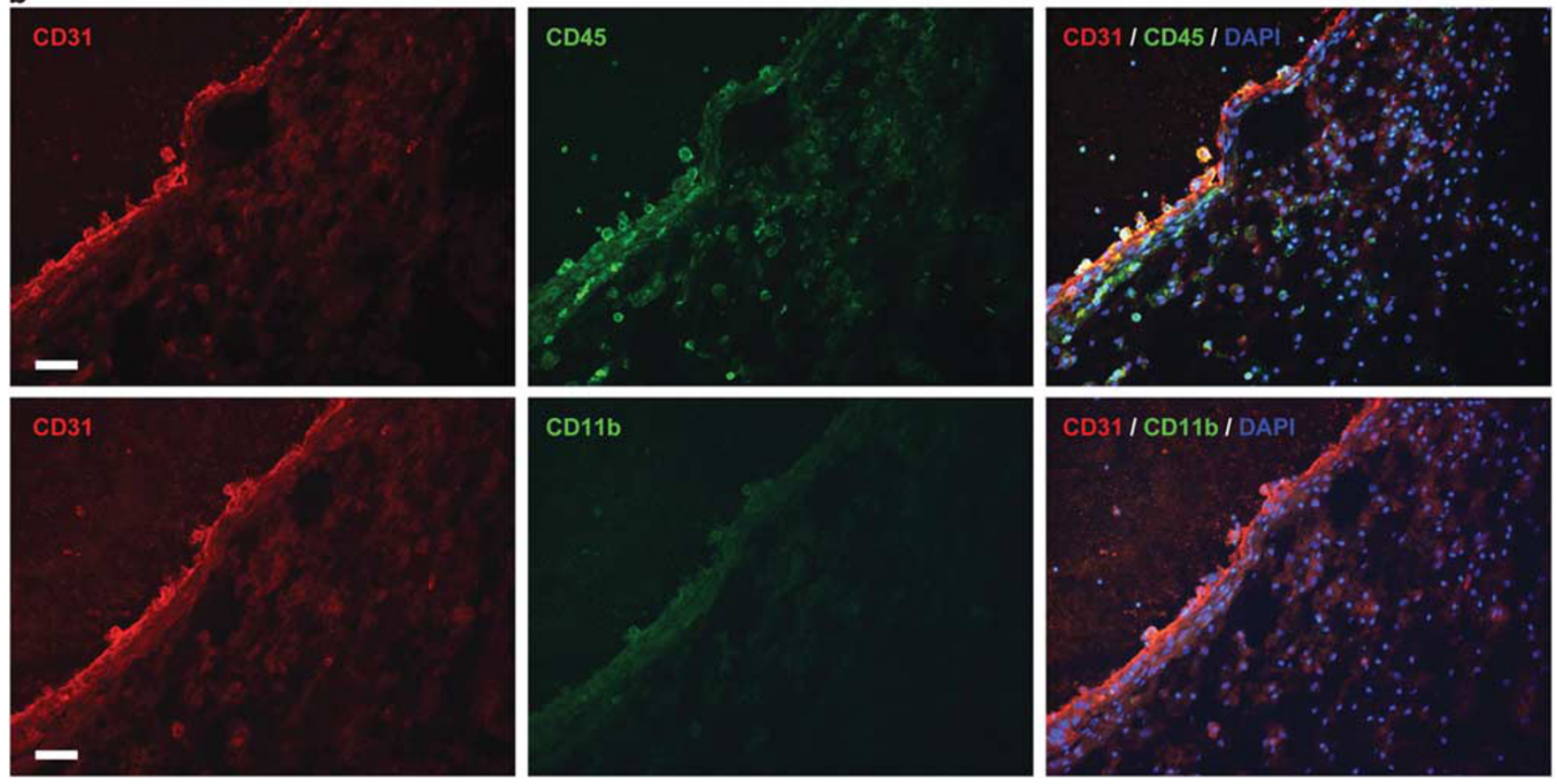

CD11b

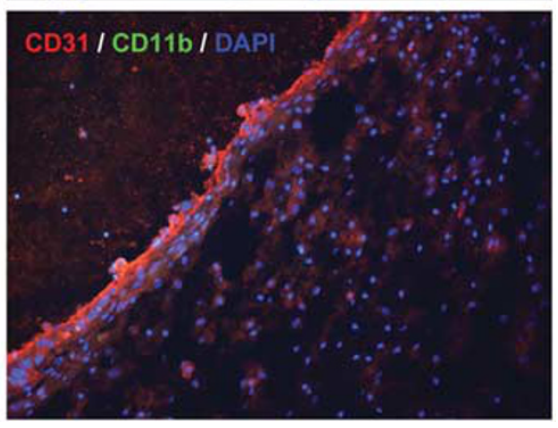


lack of endothelial differentiation in this area at this time point. Immuno-co-localization with CD31 and Ki67 (second row) revealed Ki67 positivity in mononuclear immune cell nuclei with none being detected in endothelial cell nuclei in peri-implant or implant areas (circled endothelial cells) at this time point. Survivin (third row) displayed a similar
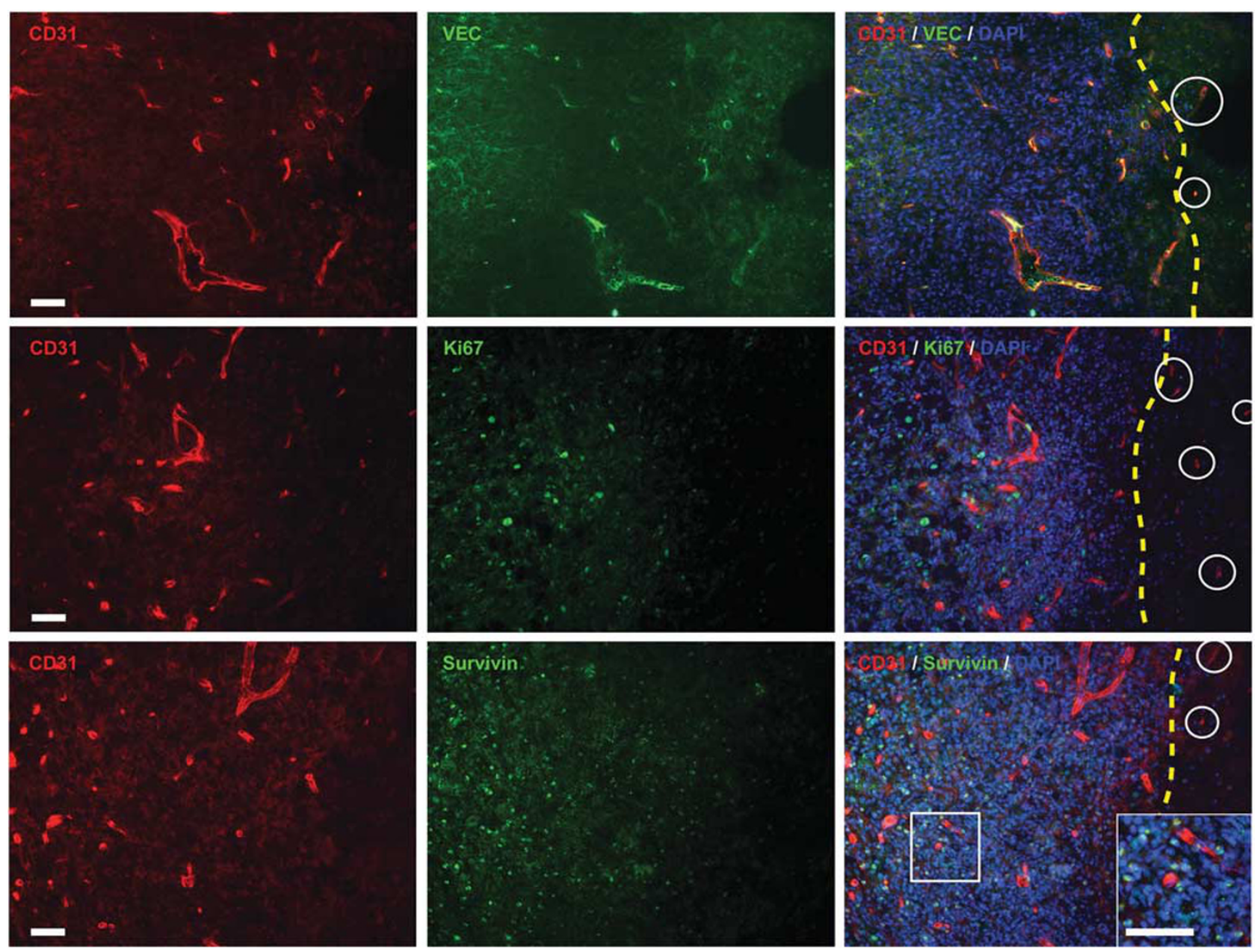

Figure 3 Dynamic Survivin and Hippo pathway component expression in the peri-implant and implant areas at the 2-week time point. Hydrogel implants after 2 weeks. Immunofluorescence staining for CD31 (red, left column), VE-cadherin (VEC) (green, middle column, top row), merged CD31/VEC/ DAPI (right column, top row), Ki67 (green, middle column, second row), merged CD31/Ki67/DAPI (right column, second row), Survivin (green, middle column, third row), merged CD31/Survivin/DAPI (right column, third row), YAP (green, middle column, fourth row), merged CD31/YAP/DAPI (right column, fourth row), phospho-YAP (P-YAP) (green, middle column, fifth row), merged CD31/P-YAP/DAPI (right column, fifth row), GLU-T1 (green, middle column, sixth row), and merged CD31/GLUT-1/DAPI (right column, sixth row). Yellow dashed lines represent the borderline between hydrogel and granulation tissue. Circles denote CD31-positive endothelial cells in the implant areas. Inserts in merged panels illustrate that Survivin-positive proliferating cells infiltrated into the perivascular area as did occasional Ki67-positive cells. YAP was localized in nuclear and cytoplasmic localizations in the microvascular endothelial cells as well as in the cytoplasm of mononuclear-infiltrating cells around blood vessels (insert, right panel, fourth row). P-YAP was localized in the cytoplasm of newly formed blood vessels as well as infiltrating mononuclear cells (Insert, right panel, fifth row). GLUT-1 was found to be colocalized with CD31 in endothelial cells (insert, right panel, bottom row). Scale bars, $50 \mu \mathrm{m}$.

Figure 2 Dynamic Inflammatory cell recruitment and angiogenesis in peri-implant tissue and implants over time. (a) Immunofluorescence imaging of CD31 (red, left column), CD45 (green, middle column, top row), merged CD31/CD45/DAPI (right column, top row), CD11b (green, middle column, second row), merged CD31/CD11b/DAPI (right second row from top), Mac3 (green, middle bottom row), and merged CD31/Mac3/DAPI (right column, third row) in hydrogel implants and surrounding tissues following 2-week implantations. Yellow dashed lines represent the borderline between hydrogel and surrounding granulation tissue. Inserts in merged panels illustrate the infiltration of CD45-CD11b-Mac3-positive mononuclear inflammatory cells in the peri-implant areas. Scale bars, $50 \mu \mathrm{m}$. (b) Hydrogel implants after 4-week implantations. Immunofluorescence imaging of CD31 (red, left column), CD45 (green, middle column, top row), merged CD31/CD45/DAPI (right column, top row), CD11b (green, middle second row from top), and merged CD31/ CD11b/DAPI (right second row from top). There were few CD45- and CD11b-positive mononuclear inflammatory cells throughout the fibrous granulation tissue scaffolding separating the dilated vascular structures. Scale bars, $50 \mu \mathrm{m}$. 

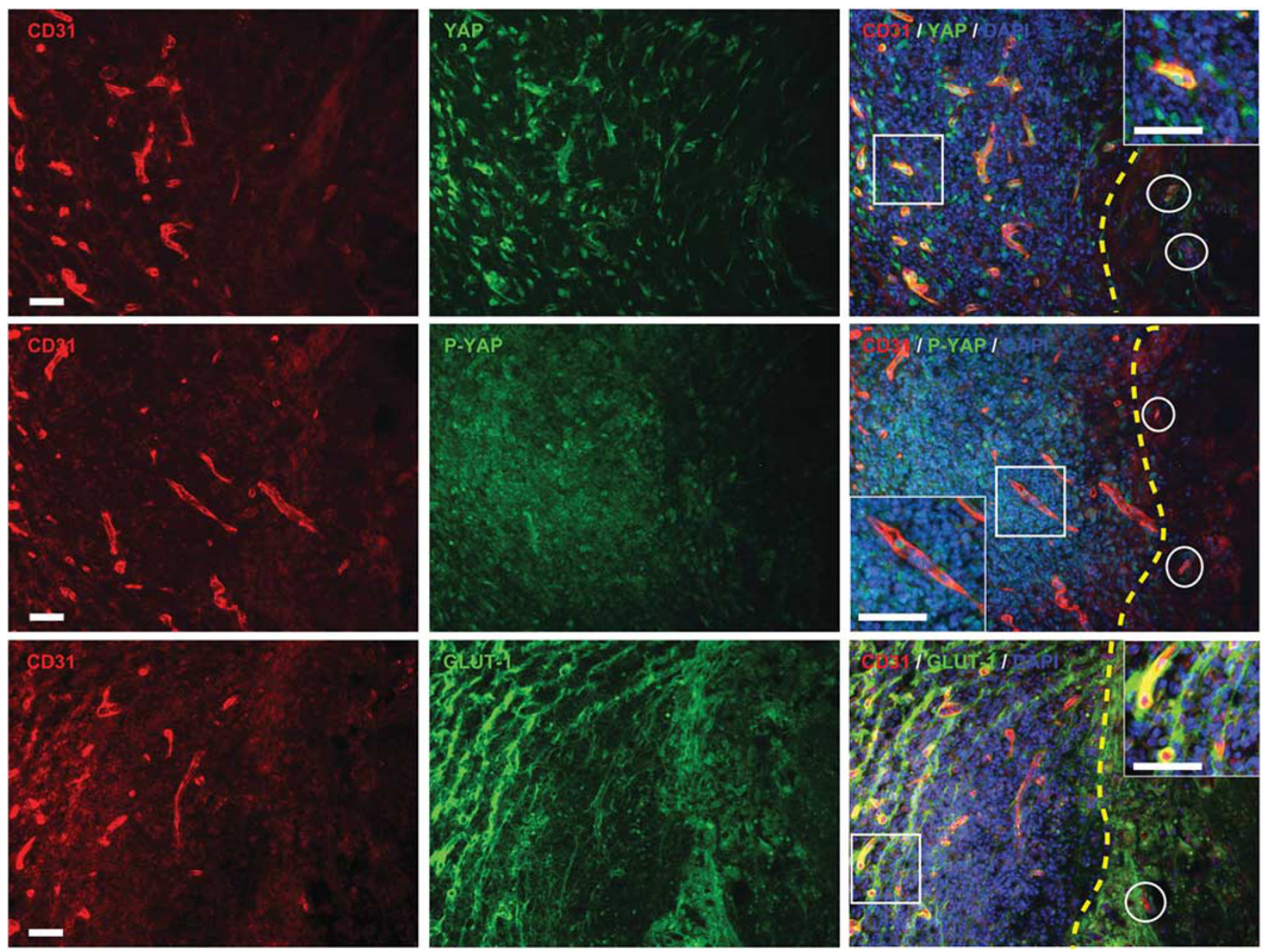

Figure 3 Continued.

localization pattern as Ki67 with modest immune cell localizations in peri-implant areas (boxed area $=$ inset) and no appreciable labeling in implant areas (circled endothelial cells). YAP localization (fourth row) appeared to be a mix of immune cell and endothelial cell localizations (boxed inset) in the peri-implant area and implant areas (circled endothelial cells). Phospho-YAP (fifth row) displayed a similar localization pattern as YAP, while having fewer positive immune cells. At the 2-week time point, immuno-co-localization with CD31 (Figure 3) and GLUT-1 (sixth row) illustrates their co-localization in peri-implant areas (left side of the yellow dashed line) but a lack of GLUT-1 in CD31-positive vessels deeper within the implant (right of the yellow dashed line, circled endothelial cells), indicative of a lack of endothelial differentiation in this area at this time point, similar to what was observed for VE-cadherin.

To further delineate the cell population(s) expressing Survivin and YAP, we double-labeled the tissues with antiCD45 and anti-Survivin or anti-YAP. As noted in Figure 4, at the 2-week time point Survivin co-localization with CD45positive mononuclear cells is minimal (first row). In contrast,
YAP co-localization with CD45-positive cells appears moderate in peri-implant areas (second row). Additionally, similar to staining illustrated in Figure 3, modest Survivin co-localization with CD45-positive cells was noted (top row). In contrast, YAP co-localization was noted with both CD31positive endothelial cells (Figure 3) as well as with CD45positive immune cells (bottom row).

At the 4-week time point, immuno-co-localization with CD31 (Figure 5, left column) and VE-cadherin (first row) illustrates their co-localization in endothelial cells lining the ectatic blood-filled spaces. Immuno-co-localization with CD31 and Ki67 (second row) revealed Ki67 positivity in two endothelial lining cells (arrows). Survivin expression (third row) was not detected at this time point (third row). Robust YAP expression appeared limited to the lining endothelial cells (inset) but absent in the dense connective tissue septa. Modest phospho-YAP expression (fifth row) also appeared limited to the lining endothelial cells (inset) but absent in the dense connective tissue septa. At the 4-week time point, immuno-co-localization of CD31 and GLUT-1 (sixth row) appears in a patchy, irregular pattern restricted to 

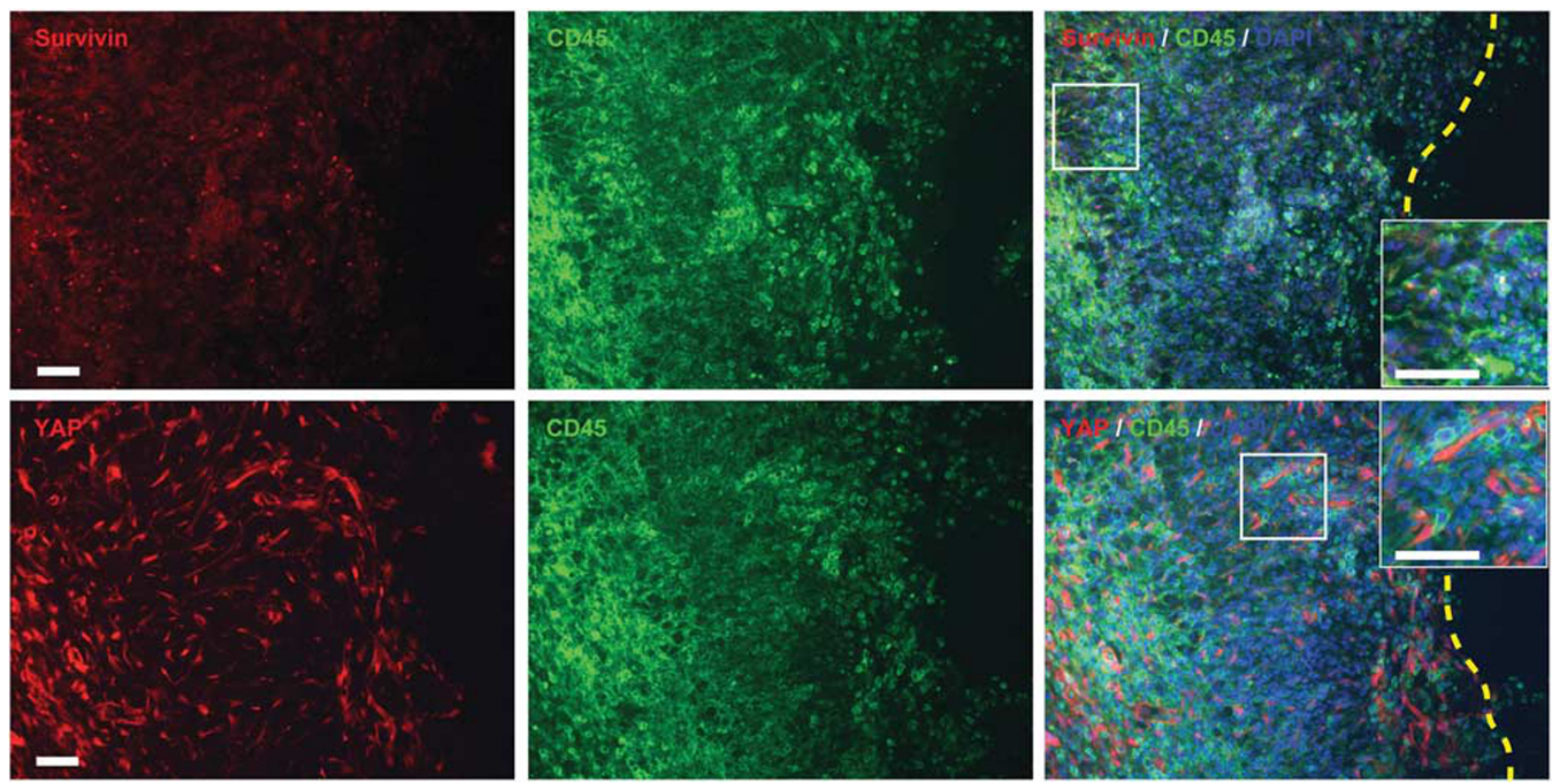

Figure 4 Survivin and Hippo pathway component expression in the mononuclear cells in the implant. Hydrogel implants after 2 weeks. Immunofluorescence staining for Survivin (red, left column, top row), CD45 (green, middle column, top row), merged Survivin/CD45/DAPI (right column, top row), YAP (red, left column, second row), CD45 (green, middle column, second row), and merged YAP/CD45/DAPI (right column, second row). Yellow dashed lines represent the borderline between hydrogel and granulation tissue. Insets in merged panels denote CD45-positive mononuclear cells in the implant areas. Scale bars, $50 \mu \mathrm{m}$.

the lining endothelial cells and more intensely in endothelial cells comprising microvessels in the dense connective tissue septa (inset).

\section{Survivin and Hippo Pathway Component Expression in Proliferative and Involuting Infantile Hemangioma Mirrors Expression Levels in Hydrogel-Endothelial Cell Implants}

Histological and immunohistochemical analyses of proliferative-phase infantile hemangiomas revealed similarities to the hydrogel-endothelial cell 2-week implants in their CD31, GLUT-1, Ajuba, Survivin, YAP, and CD45 expression levels (Figure 6). Low-power (left panel) and high-power (second panel) H\&E-stained sections revealed solid regions consisting of small vascular lumina lined by flattened endothelial cells among clusters of mononuclear cells in a scant stroma (lower half of the image), illustrative of the proliferative phase (Figures $6 \mathrm{a}_{1-3}$ ). Labeling with anti-CD31 revealed robust staining of endothelial cells lining the lumina and single endothelial cells present throughout the lower half of the image and predominately endothelial cell lining cells in the upper half of the image (left two panels, second row, lower half of the image, and the inset; Figures $6 a_{3-6}$ ). As observed for CD31 staining, GLUT-1 exhibited robust staining of endothelial cells lining the lumina and occasional single endothelial cells present throughout the image and the inset, predominately endothelial cell lining cells in the upper half of the image (left two panels, third row, and the inset; Figures $6 \mathrm{a}_{6-9}$ ). Labeling with anti-Ajuba revealed predominately nuclear and modest cytoplasmic staining of endothelial lining cells and single endothelial cells throughout the entire section (Figures $6 \mathrm{a}_{10-12}$ ). Labeling with anti-Survivin also revealed predominately nuclear staining of endothelial lining cells and single endothelial cells throughout the entire section (Figures $6 \mathrm{a}_{13-15}$ ). YAP staining was also found to be present in both the nuclei and cytoplasm of the luminal lining endothelial cells and occasional single cells in between the lumina (Figures $6 \mathrm{a}_{16-18}$ ). CD45 staining revealed robust staining of many mononuclear cells present throughout the more solid areas of the proliferative hemangioma (Figures $\left.6 \mathrm{a}_{19-21}\right)$. To support our hypothesis that the CD45-positive cells were macrophages, we stained representative infantile hemangioma lesions with anti-CD68. Examination of the stained tissue revealed significant numbers of CD68-positive cells interspersed among endothelial cells and the formed vessel segments in lesional tissue (Figures $6 \mathrm{a}_{22-24}$ ). CD3 staining revealed a very small number of $\mathrm{CD} 3$-positive cells interspersed the endothelial cells and vessel segments of lesional tissue (Figures $6 \mathrm{a}_{25-27}$ ). As expected, staining the hemangioma specimens with Ki67 revealed robust nuclear staining in the proliferative specimens (Figures $6 \mathrm{a}_{28-30}$ ).

Histological and immunohistochemical analyses of the involuted-phase infantile hemangiomas revealed similarities to the hydrogel-endothelial cell 4 -week implants in their 

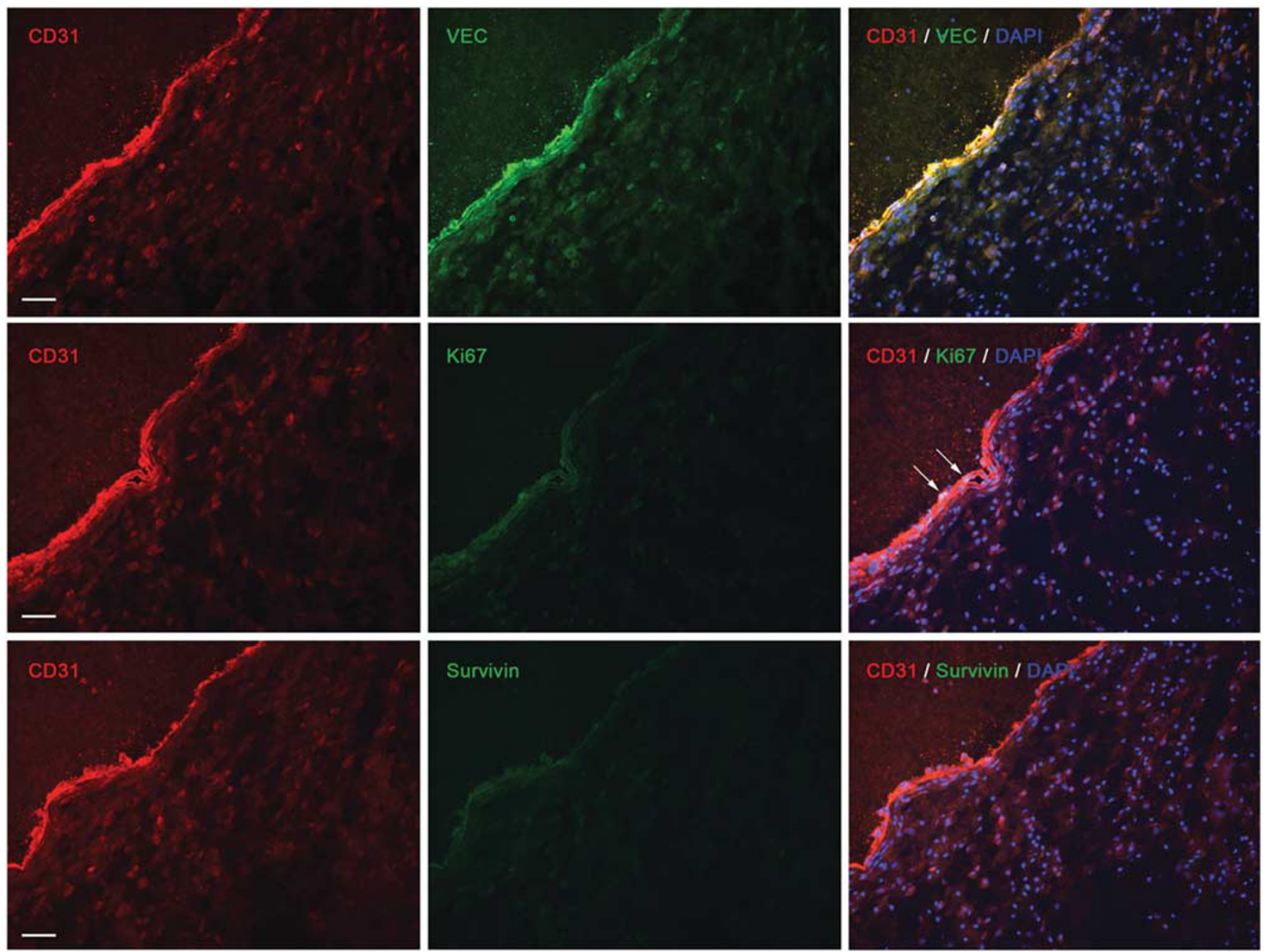

Figure 5 Dynamic Survivin and Hippo pathway component expression in the peri-implant and implant areas at the 4-week time point. In contrast to the 2-week time point, hydrogel implants after 4 weeks exhibited differential localizations and expression levels of VE-cadherin, Ki67, Survivin, Hippo pathway components (YAP and phosphor-YAP), and GLUT-1. Immunofluorescence staining for CD31 (red, left column), VE-cadherin (VEC) (green, middle column, top row), merged CD31/VEC/DAPI (right column, top row), Ki67 (green, middle column, second row), merged CD31/Ki67/DAPI (right column, second row), Survivin (green, middle column, third row), merged CD31/Survivin/DAPI (right column, third row), YAP (green, middle column, fourth row), merged CD31/YAP/DAPI (right column, fourth row), phospho-YAP (P-YAP) (green, middle column, fifth row), merged CD31/P-YAP/DAPI (right column, fifth row), GLUT-1 (green, middle column, bottom row), and merged CD31/GLUT-1/DAPI (right column, bottom row and inset) revealed modest staining of Ki67 (arrows) and Survivin, robust YAP, and moderate P-YAP staining in endothelial cells. In contrast to the 2-week implants, the 4-week implant connective tissues exhibited very modest CD31, VE-cadherin Survivin, YAP, and P-YAP staining. Interestingly. GLUT-1 was localized to the microvasculature in the connective tissue septa, colocalizing with CD31 but was absent from the endothelia lining the ectatic blood-filled spaces (left column, bottom row, and inset). Scale bars, $50 \mu \mathrm{m}$.

CD31, Survivin, YAP, CD45, and GLUT-1 expression levels. Low-power and high-power H\&E-stained sections revealed various sized vascular lumina lined by flattened endothelial cells interspersed in a dense fibrous stroma having an occasional cell interspersed throughout the stroma. (Figures $\left.6 b_{1-3}\right)$, illustrative of the involuted phase. Labeling with antiCD31 again revealed robust staining of endothelial cells lining the lumina and rare single endothelial cells interspersed throughout the stroma (Figures $6 b_{4-6}$ ). Labeling with antiGLUT-1 exhibited moderate staining of endothelial cells lining the lumina (Figures $6 \mathrm{~b}_{7-9}$ ). Labeling with anti-Ajuba revealed very modest cytoplasmic staining of endothelial lining cells (Figures $6 \mathrm{~b}_{10-12}$ ). Labeling with anti-Survivin was not detectable (Figures $6 \mathrm{~b}_{13-15}$ ), as was YAP staining (Figures $\left.6 b_{16-18}\right)$. CD45 staining in involuted hemangiomas exhibit moderate staining of only a few mononuclear cells in the connective tissue stroma and occasional positive cells in the lumina of vessels in the tumor (Figures $6 \mathrm{~b}_{19-21}$ ). Examination of the stained tissue also revealed rare CD68-positive cells interspersed among endothelial cells and formed vessel segments in lesional tissue (Figures $6 \mathrm{~b}_{22-24}$ ). CD3 staining also revealed rare $\mathrm{CD} 3$-positive cells interspersed among the vessel segments of lesional tissue (Figures $6 \mathrm{~b}_{24-27}$ ). In contrast to the proliferative specimens, staining of the involutional 

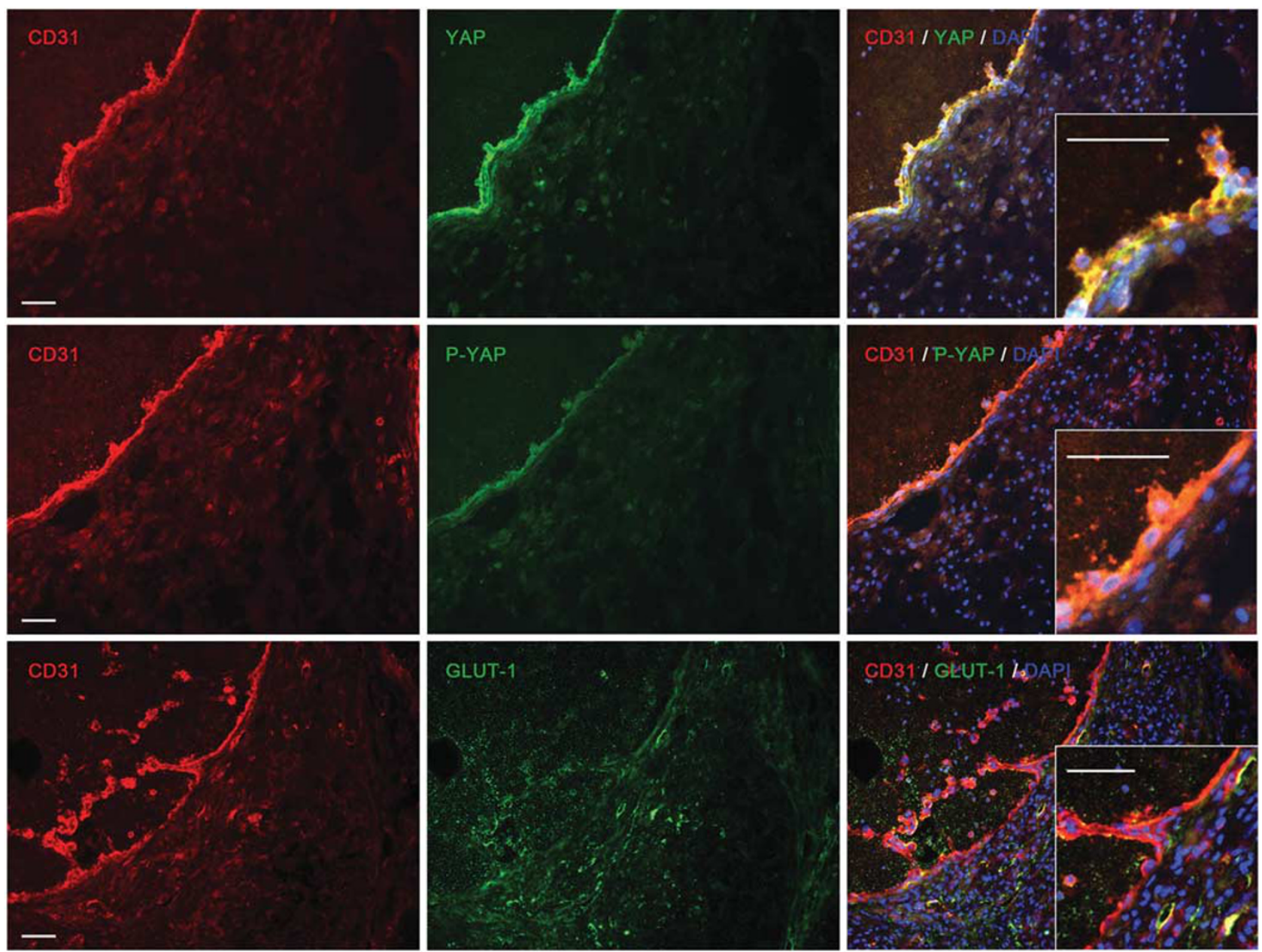

Figure 5 Continued.

hemangioma specimens with Ki67 revealed very modest nuclear staining (Figures $6 \mathrm{~b}_{28-30}$ ).

Expression Levels of Ajuba (and to a Lesser Extent YAP, Survivin, and CD45) in Infantile Hemangioma Correlate With the Proliferative and Involuting Phases of Samples Contained in our Tissue Microarray

Immunohistological analyses of representative proliferativeand involuting-phase infantile hemangiomas from our 6-mm double redundant tissue microarray using anti-Ajuba, -Survivin, -YAP, and -CD45 reagents revealed that in the proliferative phase Ki67, Ajuba, Survivin, YAP, and CD45 expression was greater than that observed in the involuted phase of infantile hemangiomas (data not shown).

This prompted analyses of the 13 proliferative- and involuting-phase infantile hemangiomas included in our tissue microarray, using anti-Ki67 (not shown), -Ajuba, -YAP, -Survivin and -CD45 reagents. This staining revealed that the staining intensities noted in the proliferative and involuted phases of infantile hemangiomas was observed as a continuum, with Ajuba intensity correlating with the degree of proliferative histology (Figure 7, first and fifth columns), with cases 1-7 correlating with a proliferative phenotype. Cases 8 and 9 displayed an intermediate staining pattern consistent with lesions in transition from proliferative to involuting phase and cases 10-13 displayed staining patterns consistent with involuting-phase hemangiomas. Although exhibiting similar expression ranges (albeit at less intense levels compared with Ajuba staining), YAP (second and sixth columns), Survivin (third and seventh columns), and CD45 (fourth and eighth columns) expression levels were also demonstrative of a continuum of staining of infantile hemangiomas from proliferative (more intense) to involuting (decreased intensity) phases.

Upon comparing our immunohistochemical- and morphological-based diagnoses (Figure 7) with the $\mathrm{H} \& \mathrm{E}$ diagnoses (Table 1, H\&E diagnosis), we found agreement with all but two cases ( 3 and 7 ) in which our immunochemical data placed them in the proliferative phase (Table 1, Surv/ Hippo analysis). Additionally, our analysis placed cases 8 and 9 in a transitional phase, expressing areas consistent with both proliferative and involutional phases. 


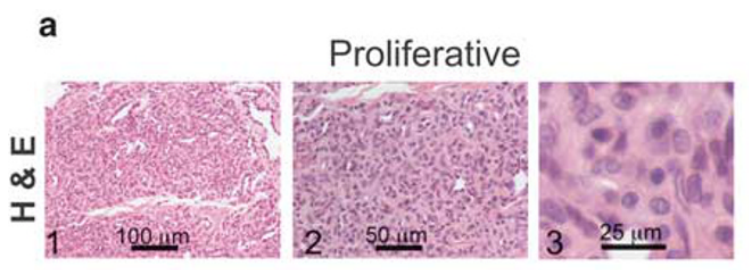

b
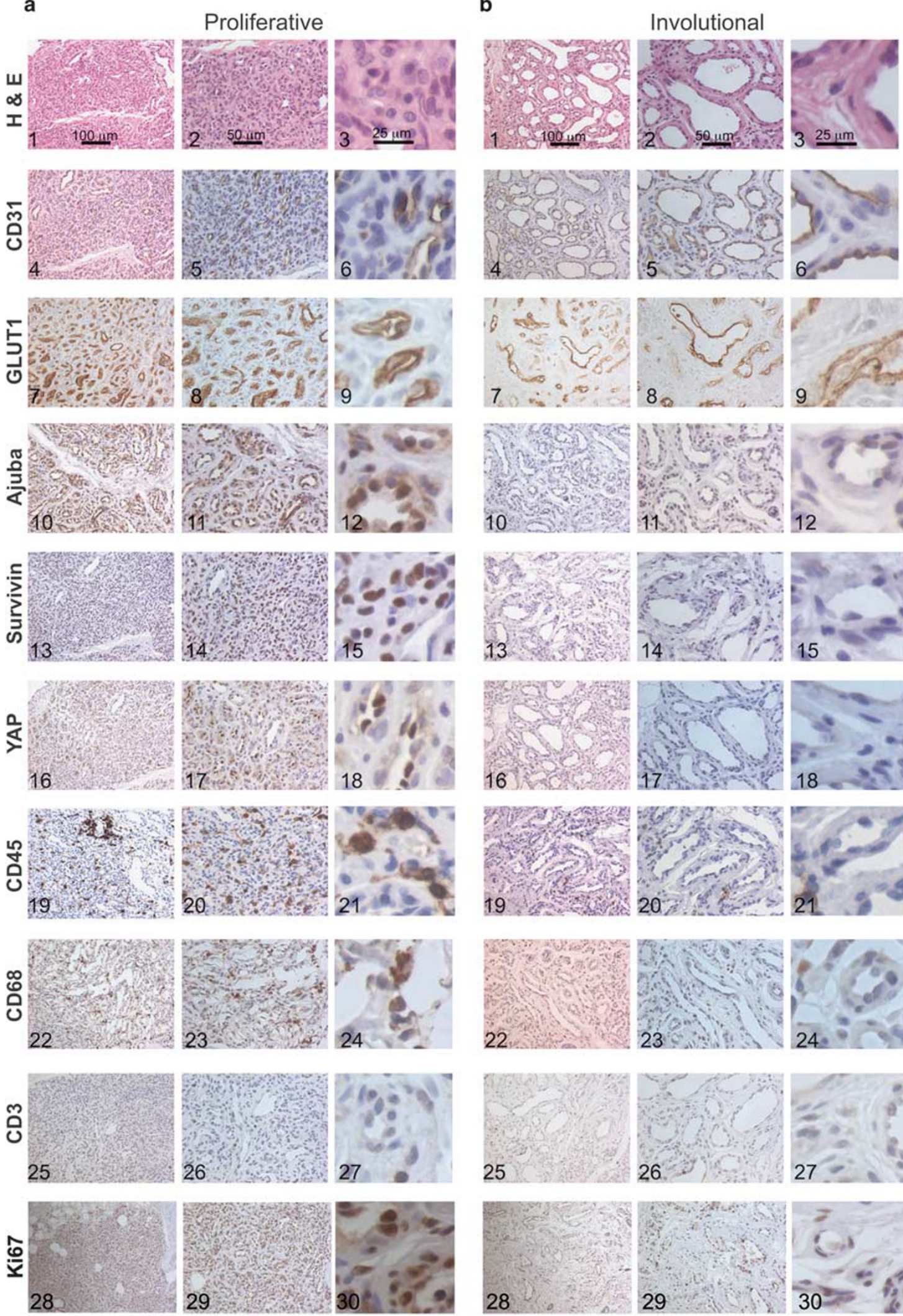
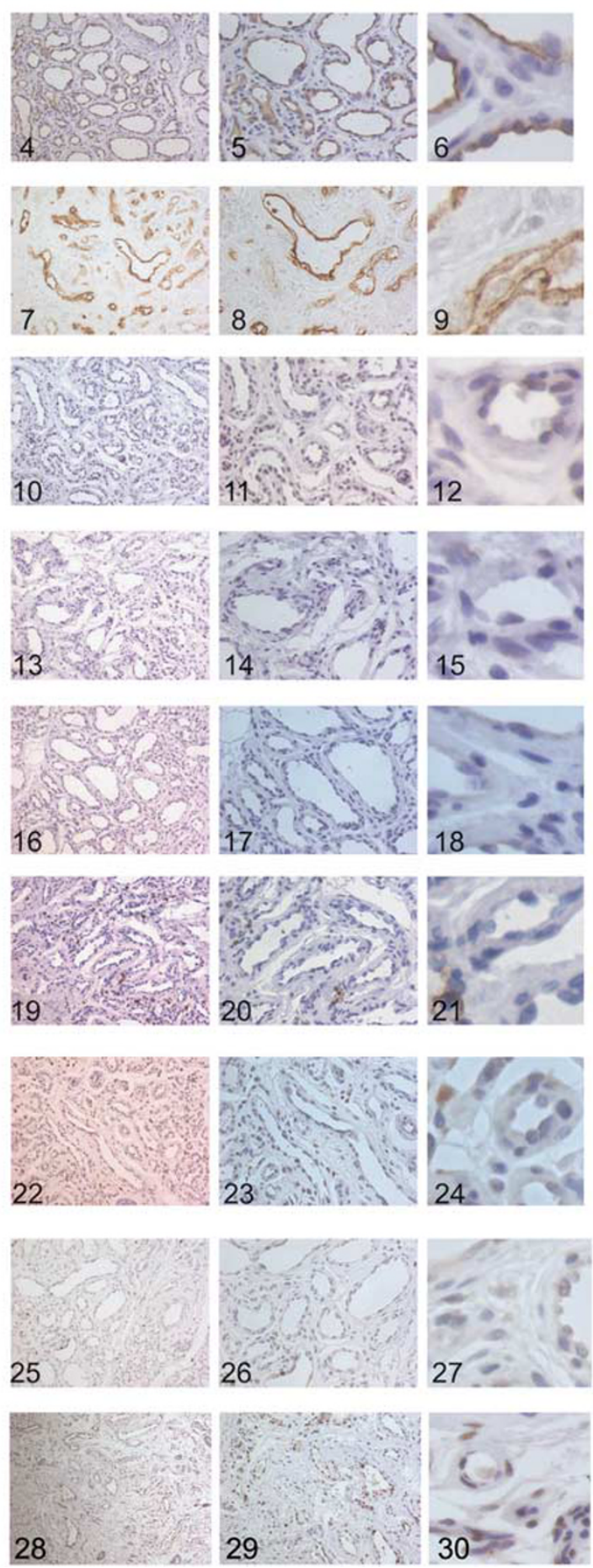

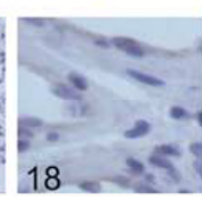



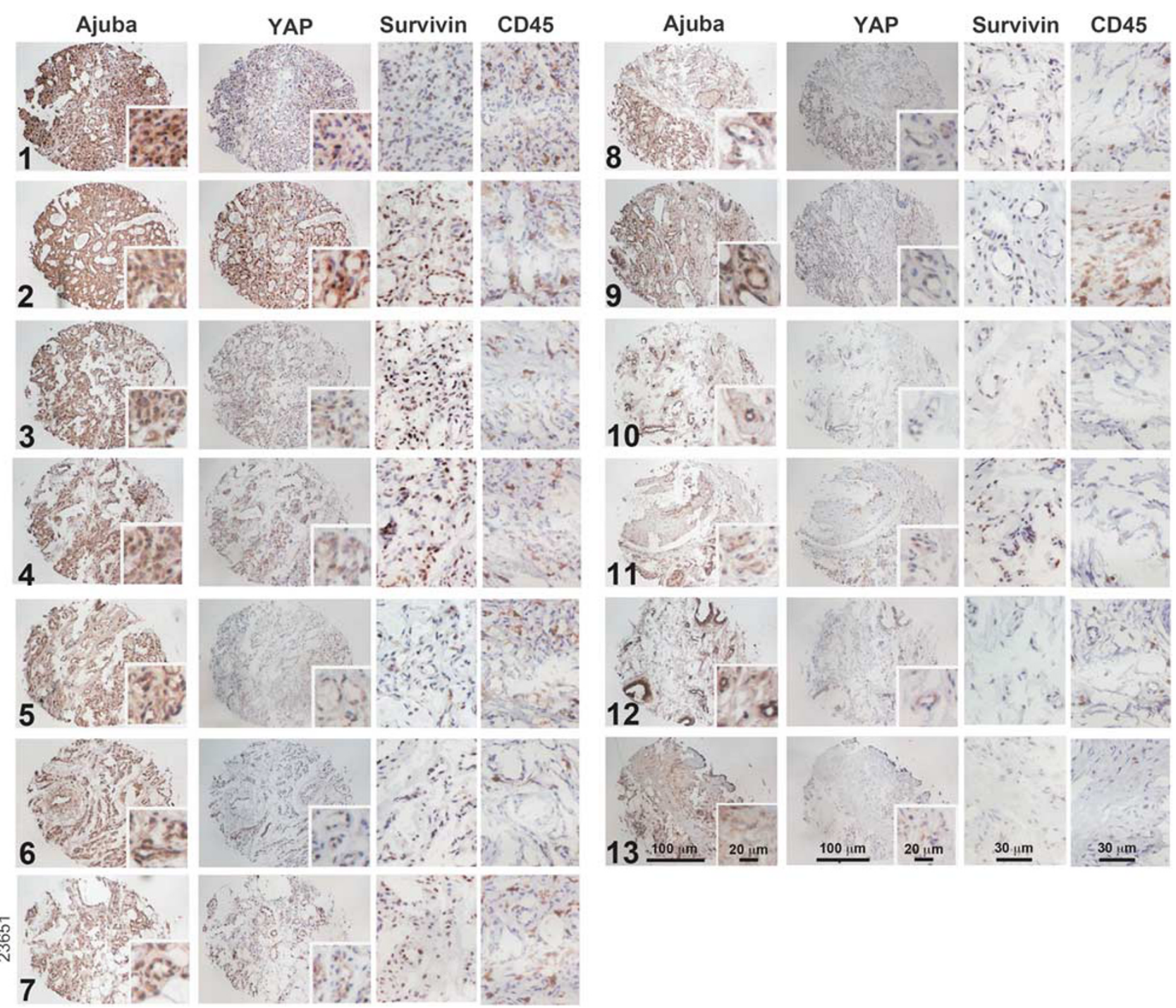

Figure 7 YAP, Ajuba, and CD45 expression levels in 13 proliferative and involuting-phase infantile hemangioma samples contained in our vascular tumor tissue microarray. Infantile hemangiomas stained for Ajuba (first column, YAP (second column), Survivin (third column), and CD45 (fourth column) were ranked according to Ajuba intensity (1-13), with (1-7) scored as proliferative and (8 and 9) scored as intermediate and have features of both proliferating and involuting hemangiomas and (10-13) scored as involuting infantile hemangiomas according to their Ajuba intensity in our double redundant tissue array. Although staining intensities of YAP, Survivin, and CD45 are much lighter, they reflect similar degrees of staining intensity and are consistent with the Ajuba scoring. Scale bars: first and second columns $=100 \mu \mathrm{m}$, insets $=20 \mu \mathrm{m}$; third and fourth columns $=30 \mu \mathrm{m}$.

Figure 6 Immunofluorescence analysis of proliferative (a) and involutional (b) infantile hemangiomas. (a) H\&E stains of proliferative lesions exhibit solid areas comprised of single endothelial cells and endothelial cells forming small vascular structures, interspersed with mesenchymal and immune cells

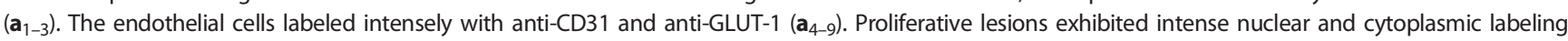
with anti-Ajuba $\left(\mathbf{a}_{10-12}\right)$ and robust nuclear labeling with anti-Survivin $\left(\mathbf{a}_{13-15}\right)$ and YAP $\left(\mathrm{A}_{16-18}\right)$. In addition, the proliferative lesions exhibited labeling with anti-CD45 and -CD68, consistent with macrophages $\left(\mathbf{a}_{19-24}\right)$. Additionally, these lesions exhibited occasional labeling with anti-CD3, indicative of $T$ lymphocytes $\left(\mathbf{a}_{25-27}\right)$. Proliferative lesions exhibited intense nuclear and labeling with anti-Ki67 $\left(\mathbf{a}_{28-30}\right)$. Scale bars: $\mathbf{a}_{1,4,7,10,13,16,19,22,25,28}=100 \mu$ m;

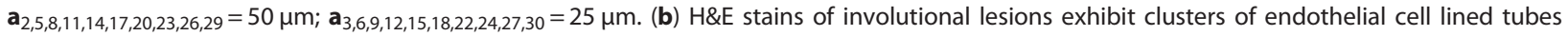
interspersed in a dense fibrous mesenchyme comprised of occasional microvascular vessels and fibroblastic cells ( $\left.\mathbf{b}_{1-3}\right)$. Endothelial cells labeled intensely with anti-CD31 and anti-GLUT-1 ( $\left.\mathbf{b}_{4-9}\right)$. In contrast to the proliferative lesions, the involutional lesions exhibited very modest to undetectable

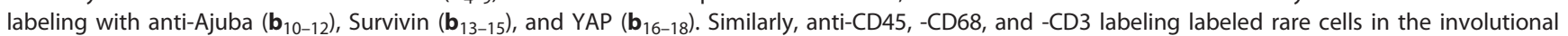
lesions ( $\left.\mathbf{b}_{19-27}\right)$. In contrast to the proliferative lesions, the involutional lesions exhibited modest nuclear labeling with anti-Ki67 ( $\left.\mathrm{B}_{28-30}\right)$. Scale bars: $\mathbf{b}_{1,4,7,10,13,16,19,22,25,28}=100 \mu \mathrm{m} ; \mathbf{b}_{2,5,8,11,14,17,20,23,26,29}=50 \mu \mathrm{m} ; \mathbf{b}_{3,6,9,12,15,18,22,24,27,30}=25 \mu \mathrm{m}$. 


\section{DISCUSSION}

Infantile hemangiomas are common benign tumors in childhood, being diagnosed in as many as $10 \%$ of children aged $<1$ year, with increased incidence in premature infants and females. ${ }^{21-23}$ Hemangiomas share a common feature of robust proliferation at the initial stages during their development followed by a regressive phase and scar formation. ${ }^{22}$ The majority of these lesions do not require any intervention. However, facial lesions and lesions of large size sometimes do require treatment that has included systemic steroids, immune regulators, Interferon alpha, propranolol, radiation, laser treatment, and surgery, ${ }^{22,24-27}$ all having some potential side effects. Several underlying mechanisms involved in the development of infantile hemangiomas have been proposed and discussed, although a definitive mechanism is still elusive. $^{23,26,28-31}$ Evidence has been accrued suggesting that the placenta may be related to infantile hemangioma in that these lesions have been associated with mothers who have undergone chorionic villus sampling. Although interesting, investigators have not observed maternal-fetal chimerism and that the genotype of the lesions is that of the child, not the mother. Another theory also involving the placenta is based on the observations that tumor-derived secreted substances can elicit changes in specific organs and tissues that predispose them to metastases ${ }^{32,33}$ (in this case, the fetal placenta or a chorangioma would be the tissue source of the putative substances and the hemangioma precursor cells). In addition, the cell of origin of hemangiomas has been postulated to be the result of a mutation(s) in a precursor cell as investigators have determined that cells isolated from hemangioma tissue have exhibited clonality. ${ }^{23,34}$ Additionally, local and systemic perturbations in the individual during infancy may predispose to the development of hemangiomas. ${ }^{35}$ Specifically, hypoxia has been suggested as a driver leading to upregulation of transcription factors and chemokines, which can elicit recruitment of endothelial progenitor cells and hemangioma development. ${ }^{35}$ Although contributing to our understanding of hemangiomas, no single theory adequately explains all pathobiology of these lesions and highlights the complexity of these lesions. ${ }^{27,30,31}$

Our findings that the kinetics of our three-dimensional subcutaneous implanted hydrogel-endothelial cell cultures mimicked the proliferative and involutional phases of human infantile hemangiomas prompted us to further characterize and compare the cellular composition of our murine model and the human counterpart. Surprisingly, we noted that many of the cells comprising the murine implants at the 2-week time point were not endothelial but expressed the markers of mononuclear cells, namely CD45, CD11b, and Mac3. The inflammation we observed appears to be related to the cell types used. When these same materials are used with other cell types, we do not see a similar infiltration of inflammatory cells. $3,10,12,17,18$ Analysis of the proliferative-phase human infantile hemangiomas also revealed the presence of CD45and CD68-positive cells interspersed among single and lumen-forming endothelia. When the murine implants were assessed for the presence of CD45, CD11b, and Mac3 at the 4-week time point, a significantly reduced number of these cells were noted. Similarly, analysis of the involutional-phase human infantile hemangiomas revealed a significantly reduced presence of CD45- and CD68-positive cells interspersed among lumen-forming endothelia. These similarities between the kinetics of our murine subcutaneous implants and the human biopsies suggests a possible dynamic, temporal role for immune cells in the proliferative phase of infantile hemangiomas, either as an initiating cell in the proliferative phase and/or as a cell involved in downregulating the proliferative response or inhibiting proliferative signals following an initiating event. Precedence for such a role for monocytes/macrophages has been well documented during the inflammatory process ${ }^{36,37}$ as well as during developmental/regenerative processes ${ }^{36,38,39}$ and has been observed and documented in infantile hemangiomas. ${ }^{27,40,41}$ Our finding of the increased numbers of CD45+, CD58+ cells in proliferating infantile hemangiomas compared with involuting infantile hemangiomas provides additional evidence suggesting the potential importance of these cells and their interactions with endothelial cells in the biological behavior of this entity. Additionally, this observation adds another potential mechanism explaining, in part, yet another aspect of local and/or systemic perturbations in the individual during infancy (which may evoke an immune response) and thus may predispose to the development of hemangiomas and/or their involution. The presence of specific myeloid cells has been postulated as having roles in the proliferative phase and involution noted in the life cycle of infantile hemangiomas. ${ }^{27,41}$ It has been reported that treatment with an immune regulator, imiquimod cream, caused accelerated regression of proliferative hemangiomas, ${ }^{27}$ consistent with a role of immune cells in the modulation of the proliferative phase of these lesions. Regarding modulation of the involutional phase and subsequent scarring or fibrosis of infantile hemangiomas, other than the association of involution with age there has been no mechanism demonstrated. ${ }^{42}$

In addition, in light of our recent findings demonstrating a role for Survivin and Hippo pathway components as modulators of endothelial and hemangioendothelioma proliferation and apoptosis in vitro, ${ }^{9,14}$ we embarked upon an assessment of components of these pathways in our murine model and in patient samples of infantile hemangioma. Interestingly, we found early (2 week) implants and proliferating infantile hemangiomas exhibited more robust Survivin, YAP, and Ajuba staining than late (4 week) implants and involutional infantile hemangiomas. Of these molecules, Ajuba (an inhibitor of LATS/12, a kinase that phosphorylates YAP, targeting it to the proteosome, reducing Hippo activity, and eliciting increased proliferation and reduced apoptosis ${ }^{9,14}$ ), gave the best correlation with the phase of the hemangiomas. Interestingly, Survivin has been examined in canine vascular tumors $^{43}$ and found not to be increased in hemangiomas. 
However, the data shown in this report ${ }^{43}$ are illustrative of an involutional-phase hemangioma, which is consistent with our results. Overall, our data are consistent with the possibility that the Survivin and Hippo pathways are modulators of proliferation and apoptosis in both the murine and human entities and that inhibition of either or both of these pathways (inhibition of Survivin activity and/or neutralization of Hippo pathway inhibitors) may be potential therapeutic targets. ${ }^{44}$ As we have recently noted, a small-molecule Survivin inhibitor YM155, exhibiting modest side effects and currently under study in clinical trials for solid tumors, is an effective modulator of murine hemangioendothelioma (EOMA) cells in culture while having no appreciable effect on wild-type endothelial cells in vitro. ${ }^{9}$ This small molecule inhibits Survivin expression by interfering with Sp1 binding to the Survivin promoter. ${ }^{45}$ Inhibiting Survivin expression was found to induce the expression of VE-cadherin and CD31, facilitating adherens junction formation, thus enhancing contact inhibition-mediated reduction of proliferation, inhibiting Ajuba expression, resulting in activation of the Hippo pathway, reducing nuclear YAP levels, reducing proliferation, and increasing apoptosis. ${ }^{9}$ Thus we speculate that selective inhibition of Survivin and/or inhibitors of the Hippo pathway may have potential as another beneficial treatment for proliferating infantile hemangiomas that have significant pathological potential, reducing subsequent scar formation and disfigurement as well as for other more aggressive vascular tumors. Finally, our murine model may have potential as a model that will allow for the elucidation of the underlying mechanisms driving the proliferative and involutional phases of this entity and as a modest through-put screen for compounds targeting these and other signaling pathways, leading to human phase trials.

\section{ACKNOWLEDGMENTS}

This work was supported in part by an unrestricted gift from Joseph and Lucille Madri to JAM; a NIH Director's New Innovator Award No. DP20D007338 to EL; as well as a grant-in aid from the JSPS (Japan Society for the Promotion of Science) research fellowship for young scientists (No. 23-1154), a JSPS postdoctoral fellowship for research abroad (No. 513), and a Uehara Memorial Foundation postdoctoral fellowship for study abroad (No. 201230024) to MT.

\section{DISCLOSURE/CONFLICT OF INTEREST}

The authors declare no conflict of interest.

1. Atesok K, Fu FH, Wolf MR et al. Augmentation of tendon-to-bone healing. J Bone Joint Surg Am 2014;96:513-521.

2. Battiston KG, Cheung JW, Jain $D$ et al. Biomaterials in co-culture systems: towards optimizing tissue integration and cell signaling within scaffolds. Biomaterials 2014;35:4465-4476.

3. Rauch MF, Michaud $\mathrm{M}, \mathrm{Xu} \mathrm{H}$ et al. Co-culture of primary neural progenitor and endothelial cells in a macroporous gel promotes stable vascular networks in vivo. J Biomater Sci Polym Ed. 2008;19:1469-1485.

4. Naito Y, Williams-Fritz M, Madri JA et al. 2012Characterization of the natural history of extracellular matrix production in tissue engineered vascular grafts during neovessel formation. Cells Tissues Organs. 2012;195:60-72.

5. Squinto SP, Madri JA, Kennedy $S$ et al. The ENCEL system: a somatic cell protein delivery system. In Vivo. 1994;8:771-780.
6. Harding MJ, Lepus C.M, Gibson T et al. Human fetal hepatoblasts implanted within a vascularized extrahepatic matrix survive long-term and support hepatitis $C$ virus infection following engraftment in immunodeficient mice. PLoS One 2010;5:e9987.

7. Madri JA, Pratt BM, Tucker AM. Phenotypic modulation of endothelial cells by transforming growth factor-beta depends upon the composition and organization of the extracellular matrix. J Cell Biol 1988;106: 1375-1384.

8. Madri JA, Williams SK. Capillary endothelial cell cultures: phenotypic modulation by matrix components. J Cell Biol 1983;97:153-165.

9. Tsuneki M, Madri JA. Adhesion molecule-mediated hippo pathway modulates hemangioendothelioma cell behavior. Mol Cell Biol 2014;34:4485-4499.

10. Ford MC, Bertram JP, Hynes SR et al. A macroporous hydrogel for the coculture of neural progenitor and endothelial cells to form functional vascular networks in vivo. Proc Natl Acad Sci USA 2006;103: 2512-2517.

11. $\mathrm{Xu} \mathrm{W}, \mathrm{Hu} X, \mathrm{Pan} \mathrm{W}$. Tissue engineering concept in the research of the tumor biology. Technol Cancer Res Treat 2014;13:149-159.

12. Rauch M, Hynes S.R, Bertram J et al. Engineering angiogenesis following spinal cord injury: a coculture of neural progenitor and endothelial cells in a degradable polymer implant leads to an increase in vessel density and formation of the blood-spinal cord barrier. Eur J Neuroscinece 2009;29:132-145.

13. Williams C, Rauch M.F, Michaud M et al.,. Neural progenitor cells control in vivo formation of engineered functional vascular networks. PLoS One 2012;7:e53208.

14. Tsuneki M, Madri J.A. CD44 regulation of endothelial cell proliferation and apoptosis via modulation of CD31 and VE-cadherin expression. J Biol Chem 2014;289:5357-5370.

15. Flynn K, Michaud M, Madri J.A. CD44 deficiency contributes to enhanced experimental autoimmune encephalomyelitis: a role in immune cells and vascular cells of the blood brain barrier. Am J Pathol 2013;182:1322-1336.

16. Flynn KM, Michaud M, Canosa $\mathrm{S}$ et al. CD44 regulates vascular endothelial barrier integrity via a PECAM-1 dependent mechanism. Angiogenesis 2013;16:689-705.

17. Royce Hynes S, McGregor L.M, Ford Rauch $M$ et al. Photopolymerized poly(ethylene glycol)/poly(L-lysine) hydrogels for the delivery of neural progenitor cells. J Biomater Sci Polym Ed 2007;18:1017-1030.

18. Williams $C$, Ford Rauch $M$, Michaud $M$ et al. Short term interactions with long term consequences: modulation of chimeric vessels by neural progenitors. PLoS One 2012;7:e53208.

19. Hertz J, Robinson R, Valenzuela D.A et al. A tunable synthetic hydrogel system for culture of retinal ganglion cells and amacrine cells. Acta Biomater 2013;9:7622-7629.

20. Pinter $\mathrm{E}$, Barreuther M, Lu T et al. Platelet-endothelial cell adhesion molecule-1 (PECAM-1/CD31) tyrosine phosphorylation state changes during vasculogenesis in the murine conceptus. Am J Pathol 1997;150: 1523-1530.

21. Goelz R, Poets C.F. Incidence and treatment of infantile haemangioma in preterm infants. Arch Dis Child Fetal Neonatal Ed 2014;100: F85-F91 Epub ahead of print.

22. Huoh KC, Rosbe K.W. Infantile hemangiomas of the head and neck. Pediatr Clin North Am 2013;60:937-949.

23. Barnés CM, Christison-Lagay E.A, Folkman J. The placenta theory and the origin of infantile hemangioma. Lymphat Res Biol 2007;5:245-256.

24. Zheng JW, Zhang $L$, Zhou $Q$ et al. A practical guide to treatment of infantile hemangiomas of the head and neck. Int J. Clin Exp Med 2013;6:851-860.

25. Stiles JM, Amaya C, Rains $S$ et al. Targeting of beta adrenergic receptors results in therapeutic efficacy against models of hemangioendothelioma and angiosarcoma. PLoS One 2013;8:e60021.

26. Lo K, Mihm M, Fay A. Current theories on the pathogenesis of infantile hemangioma. Semin Ophthalmol 2009;24:172-177.

27. Sun Z-J, Zhao. Y.F, Zhang. W.F. Immune response: a possible role in the pathophysiology of hemangioma. Med Hypotheses 2007;68:353-355.

28. Nguyen VA, Kutzner $\mathrm{H}$, Fürhapter $\mathrm{C}$ et al. Infantile hemangioma is a proliferation of LYVE-1-negative blood endothelial cells without lymphatic competence. Mod Pathol 2006;19:291-298.

29. North $P E$, Waner $M$, Buckmiller $L$ et al. Vascular tumors of infancy and childhood: beyond capillary hemangioma. Cardiovasc Pathol 2006;15: 303-317. 
30. Luu M, Frieden I.J. Haemangioma: clinical course, complications and management. Br J Dermatol 2013;69:20-30.

31. Chen TS, Eichenfield L.F, Friedlander S.F. Infantile hemangiomas: an update on pathogenesis and therapy. Pediatrics 2013;131:99-108.

32. Padua D, Massague J. Roles of TGFbeta in metastasis. Cell Res 2009;19: 89-102.

33. Gupta GP, Massague J. Platelets and metastasis revisited: a novel fatty link. J Clin Invest 2004;114:1691-1693.

34. Bischoff J. Monoclonal expansion of endothelial cells in hemangioma: an intrinsic defect with extrinsic consequences? Trends Cardiovasc Med 2002;12:220-224.

35. Chang El, Chang El, Thangarajah $\mathrm{H}$ et al. Hypoxia, hormones, and endothelial progenitor cells in hemangioma. Lymphat Res Biol 2007;5: 237-243.

36. Hibino NYT, Duncan D.R, Rathore A et al. A critical role of macrophages on neovessel formation and the development of stenosis in tissue engineered vascular grafts. FASEB J 2011;25:4253-4263.

37. Novak ML, Koh TJ. Phenotypic transitions of macrophages orchestrate tissue repair. Am J Pathol 2013;183:1352-1363.

38. Spiller KL, Anfang R.R, Spiller J et al. The role of macrophage phenotype in vascularization of tissue engineering scaffolds. Biomaterials 2014;35:4477-4488.
39. Baer C, Squadrito ML, Iruela-Arispe $\mathrm{ML}$ et al. Reciprocal interactions between endothelial cells and macrophages in angiogenic vascular niches. Exp Cell Res 2013;319:1626-1634.

40. Nguyen VA, Fürhapter C, Romani N et al. Infantile hemangioma is a proliferation of beta 4-negative endothelial cells adjacent to HLA-DRpositive cells with dendritic cell morphology. Hum Pathol 2004;35: 739-744.

41. Ritter MR, Reinisch J, Friedlander S.F et al. Myeloid cells in infantile hemangioma. Am J Pathol 2006;168:621-628.

42. Couto RA, Maclellan R.A, Zurakowski D et al. Infantile hemangioma: clinical assessment of the involuting phase and implications for management. Plast Reconstr Surg 2012;130:619-624.

43. Murakami M, Sakai $\mathrm{H}$, Kodama A et al. Expression of the anti-apoptotic factors $\mathrm{Bcl}-2$ and survivin in canine vascular tumours. J Comp Pathol 2008;139:1-7.

44. Li W, Wang $\mathrm{H}$, Kuang $\mathrm{CY}$ et al. An essential role for the Id1/PI3K/Akt/ $\mathrm{NFkB}$ /survivin signalling pathway in promoting the proliferation of endothelial progenitor cells in vitro. Mol Cell Biochem 2012;363: 135-145.

45. Cheng $Q$, Ling $X$, Haller $A$ et al. Suppression of survivin promoter activity by YM155 involves disruption of Sp1-DNA interaction in the survivin core promoter. Int J Biochem Mol Biol 2012;3:179-197. 